\title{
Studies of the Mouse Foot-Pad Technique for Cultivation of Mycobacterium leprae. 4. Statistical Analysis of Harvest Data
}

\author{
W. MARK KRUSHAT, KENNETH E. SCHILLING, STANLEY A. EI)LAVITCH \\ AND LOUIS LEVY
}

Community Medicine Program and Leprosy Research Unit, Public Ilealth Service IIospital, San Francisco, California 94118, U.S.A.

\begin{abstract}
An analysis of data generated by harvests of Mycobacterium leprae from the footpads of mice is presented. Acid-fast bacteria (AFB) were randomly distributed within the circles of a counting slide in fewer than half of the preparations; the AFB were more likely to be distributed randomly in those preparations containing fewer organisms. The mean coefficient of variation

$$
100 \times \frac{\text { standard deviation }}{\text { mean }}
$$

of the number of AFB was $29 \%$ for the 3 circles on a counting slide, $60 \%$ for the 4 foot-pads normally pooled for a harvest, and $48 \%$ for harvests from 4 replicate pools of 4 to 8 foot-pads. The doubling time of $M$. leprae during logarithmic multiplication in mice averaged 10.7 days, confirming an almost identical estimate made in an earlier study by a different technique. Finally, multiplication of $M$. leprae was found to be a little slower in mice inoculated in both hind foot-pads than in mice inoculated in only one.

This analysis confirms the precision of data generated by work with Shepard's foot-pad technique. Except for the case of foot-by-foot harvests, differences among measurements equivalent in time or numbers of $\mathrm{AFB}$ to 2 doublings of $M$. leprae appear certainly to be meaningful.
\end{abstract}

\section{Introduction}

Since its description by Shepard (1960), the mouse foot-pad technique for the cultivation of Mycobacterium leprae has been applied to the study of numerous experimental and clinical problems in leprosy. Although the technique is demanding, restricting its employment to a few laboratories, the results of its applications have been useful. The data generated in these laboratories and the conclusions and hypotheses based on them are widely used by investigators and clinicians unfamiliar with the technique. Knowledge of the limitations of the technique is required if the results of experiments are to be correctly interpreted and applied. 
In earlier papers in this series, we examined the fate of inoculated $M$. leprace (Levy et al., 1974), studied the application of the mouse foot-pad technique to the measurement of the proportion of bacilli infective for mice in skin biopsy specimens obtained from leprosy patients (Levy and Murray, 1976), and measured the doubling time of $M$. leprue during logarithmic multiplication in the mouse foot-pad (Levy, 1976). In this report, we present a statistical analysis of the results of the mouse foot-pad harvest procedure.

\section{Materials and Methods}

All studies were performed on $M$. leprae harvested from the foot-pad tissues of locally-bred BALB/c mice according to the method described by Shepard (1960), and counted by means of a carefully-standardized technigue (Shepard and McRae, 1968). The $M$. leprae employed in the studies were of a number of strains; however, the great majority of the studies, including all measurements of doubling time, were performed with the strain furnished by Shepard in 1967 and used subsequently as the standard strain for most of the work of this laboratory. Most of the studies to be reported were based on data generated in the course of other work. A few were carried out primarily for the purpose of this report.

\section{Results}

This study is divided into 6 sections: (1) Distribution of acid-fast bacteria (AFB) within the "circles" of the Reich counting slide (Bellco Glass Co., Vineland, N.J., U.S.A.); (2) variation of the number of AFB among the 3 circles of a counting slide; (3) variation of the number of AFB among replicate harvests, each from a single foot-pad; (4) variation of the number of AFB among replicate harvests, each from a pool of 4 to 8 foot-pads; (5) doubling time of $M$. leprae during logarithmic multiplication in mice; and (6) comparison of multiplication of $M$. leprae in mice inoculated in both hind foot-pads (BHF) with that in mice inoculated only in the right hind foot-pad (RHF).

\section{DISTRIBUTION OF AFB WITHIN CIRCLES}

If the AFB were distributed randomly-that is, if each organism were independent of every other organism -within the circles of a counting slide, the sampling distribution of the AFB in the microscopic fields examined would be a "Poisson distribution" with a mean of $\lambda$ (Goldstein, 1964). To test whether the AFB were distributed according to a Poisson distribution, AFB were enumerated in each of 60 oil-immersion fields examined in the 3 circles on each of 20 slides, and the number was recorded field-by-field. A goodness-of-fit test using the $\chi^{2}$ statistic (Goldstein, 1964) was used to determine whether the observed distribution of $\mathrm{AFB} / \mathrm{f}$ ield corresponded with a Poisson distribution. An example of this calculation is shown in Table 1 . In this case (specimen no. 8 of Table 2), $120 \mathrm{AFB}$ were counted in 60 fields, yielding a mean $(\lambda)$ of $2.00 \mathrm{AFB} / \mathrm{field}$. The value of $\chi^{2}$ calculated in this example is smaller than the critical value, indicating that the differences between observed and expected frequencies are not inconsistent with a Poisson distribution.

In Table 2 , the values of $\lambda$ and $\chi^{2}$ are listed for each of 20 specimens, together with the corresponding degrees of freedom. Eleven of the 20 showed differences between observed and expected frequencies of the numbers of $\mathrm{AFB} /$ field greater 
TABLE

Expected and observed distributions of $A F B$ for specimen No. 8 yielding a total of $120 \mathrm{AFB} / 60$ fields $(\lambda=2.00 \mathrm{AFB} /$ field $)$

\begin{tabular}{|c|c|c|c|}
\hline \multirow{2}{*}{$\begin{array}{l}\text { No. AFB/ } \\
\text { field }\end{array}$} & & \multicolumn{2}{|c|}{ Frequency } \\
\hline & & Expected & Observed \\
\hline 0 & $60 e^{-2.0}$ & $=8.12$ & 11 \\
\hline 1 & $60\left(2.0 \mathrm{e}^{-2.0}\right)$ & $=16.24$ & 14 \\
\hline 2 & $\frac{60\left(4.0 \mathrm{e}^{-2.0}\right)}{2}$ & $=16.24$ & 14 \\
\hline 3 & $\frac{60\left(8.0 e^{-2.0}\right)}{3 !}$ & $=10.83$ & 11 \\
\hline$\geqslant 3$ & $60-51.43$ & 8.57 & 10 \\
\hline Total & & 60 & 60 \\
\hline \multicolumn{4}{|c|}{$\chi^{2}=\frac{(8.12-11)^{2}}{8.12}+\frac{2(16.24-14)^{2}}{16.24}+\frac{(10.83-11)^{2}}{10.83}+\frac{(8.57-10)^{2}}{8.57}=1.88^{*}$} \\
\hline
\end{tabular}

* The critical value of $\chi^{2}$ for $P=0.01$ with 3 degrees of freedom $=11.3$; therefore, the observations are consistent with random distribution of the AFB.

TABLE 2

$\chi^{2}$ as a function of $\lambda$ * for 20 specimens

\begin{tabular}{cccccccc}
\hline $\begin{array}{c}\text { Specimen } \\
\text { no. }\end{array}$ & $\lambda$ & DF $\dagger$ & $\chi^{2}$ & $\begin{array}{c}\text { Specimen } \\
\text { no. }\end{array}$ & $\lambda$ & DF $\dagger$ & $\chi^{2}$ \\
\hline 1 & 0.12 & 1 & 0.48 & 11 & 2.38 & 3 & $47.69 \ddagger$ \\
2 & 0.45 & 1 & $9.96 \ddagger$ & 12 & 2.42 & 4 & 1.12 \\
3 & 0.60 & 1 & 0.33 & 13 & 3.48 & 3 & $10.35 \S$ \\
4 & 0.82 & 1 & 0.89 & 14 & 4.43 & 4 & 7.83 \\
5 & 1.27 & 2 & $26.62 \ddagger$ & 15 & 5.72 & 3 & $42.63 \ddagger$ \\
6 & 1.37 & 1 & $49.28 \ddagger$ & 16 & 6.75 & 5 & $22.07 \ddagger$ \\
7 & 1.85 & 3 & $15.49 \ddagger$ & 17 & 7.47 & 5 & $36.78 \ddagger$ \\
8 & 2.00 & 3 & 1.88 & 18 & 8.20 & 1 & $55.92 \ddagger$ \\
9 & 2.02 & 3 & 2.26 & 19 & 9.88 & 4 & $1211 \ddagger$ \\
10 & 2.05 & 3 & $11.53 \S$ & 20 & 60.67 & 1 & $4095 \ddagger$ \\
\hline
\end{tabular}

* $\lambda=$ mean no. $\mathrm{AFB} /$ oil immersion field.

$\dagger$ Degrees of freedom.

$\ddagger P<0.01$.

$\S 0.01<P<0.05$. $P>0.05$ for all values of $\chi^{2}$ not footnoted.

than would be consistent with a Poisson distribution. Two additional specimens yielded $\chi^{2}$ values larger than the critical values for $P=0.05$ but smaller than those for $P=0.01$, suggesting that these were borderline cases.

The frequency distributions of the number of AFB per field of 3 representative specimens are shown in Fig. 1. The expected and observed frequencies for 


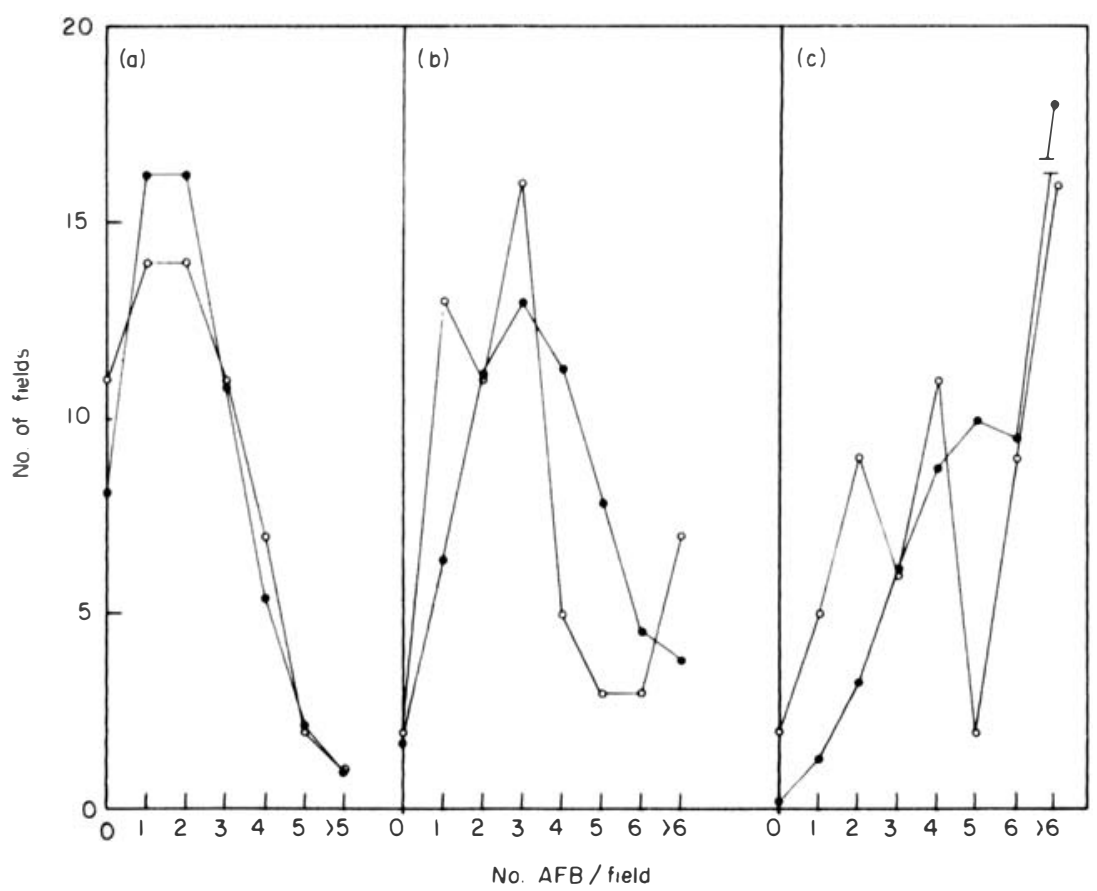

Fig. 1. Frequency distribution of the number of $\mathrm{AFB} /$ field of three representative specimens. (a) Specimen no. $8 ; \lambda=2.00, \chi^{2}=1.88, P>0.05$. (b) Specimen no. $13 ; \lambda=3.48$, $\chi^{2}=10.35,0.05>P>0.01$. (c) Specimen no. $15, \lambda=5.72, \chi^{2}=38.73, P<0.01$. The expected frequency $(\bullet)$ and $(0)$ the observed frequency.

specimen no. 8 , shown in the left-hand panel, are quite similar. The expected and observed frequencies are plotted in the center panel for specimen no. 13, which yielded a $\chi^{2}$ value of borderline significance. In this case, more than the expected numbers of fields contained $1 \mathrm{AFB}$ and more than $6 \mathrm{AFB}$, whereas the numbers of fields containing 4 or $5 \mathrm{AFB}$ were smaller than expected. The excessive number of fields observed to contain small numbers of $\mathrm{AFB}$ is even more apparent in the distribution of AFB shown in the right-hand panel from specimen no. 15, which yielded a significantly large value of $\chi^{2}$. In this case also, the number of fields containing intermediate numbers of AFB is smaller than expected.

Inspection of the data of Table 2 suggests that the AFB are less likely to be distributed according to a Poisson distribution in preparations yielding larger values of $\lambda$. This impression may be tested by plotting $\log _{10} \chi^{2}$ as a function of $\log _{10} \lambda$ for these 20 samples. The regression of $\log _{10} \chi^{2}$ on $\log _{10} \lambda$, shown in Fig. 2, has a slope significantly greater than 0 , confirming that AFB from specimens with smaller values of $\lambda$ are more likely to be randomly distributed in the circles of a counting slide than are AFB from specimens yielding larger values of $\lambda$.

\section{VARIATION OF THE NUMBER OF AFB FROM CIRCLE TO CIRCLE}

The variation of the number of AFB among the 3 circles of a counting slide was determined for the 233 slides prepared during 1975 that yielded a total of at least 


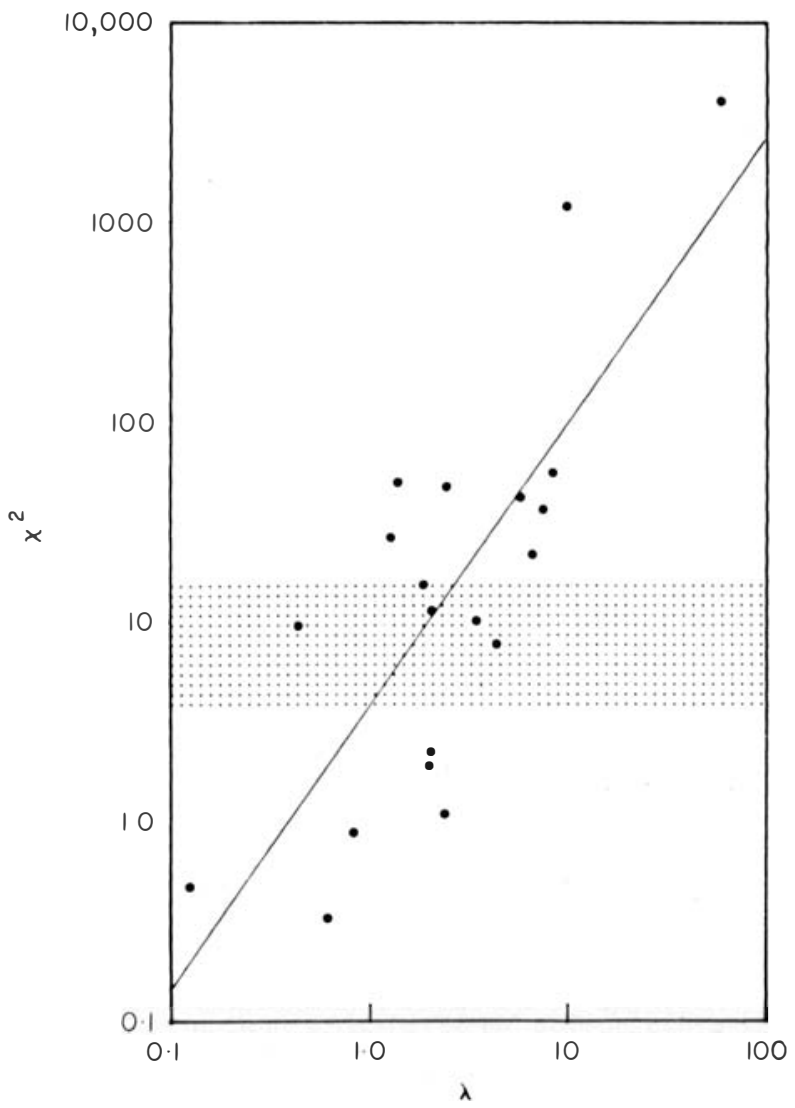

Fig. 2. $\log _{10} \chi^{2}$ as a function of $\log _{10} \lambda(\lambda=$ mean no. $A B F /$ field $)$ for 20 preparations in which the number of AFB counted in each microscopic field was compared to that expected from a Poisson distribution. The shaded area represents the region of critical values of $\chi^{2}$ for $0.05>P>0.01$ and 1 to 5 degrees of freedom. The equation of the regression line is: $\log _{10} \chi^{2}=1.14+(1.41 \pm 0.55)\left(\log _{10} \lambda-0.40\right)$; the correlation coefficient, $r=0.78$.

$50 \mathrm{AFB}$ in the 60 fields examined. For each slide, the mean and standard deviation of the number of AFB in each of the 3 circles were calculated. The variation from the mean of the 3 circles of the number of AFB counted in each of the circles is expressed as the coefficient of variation (C.V.), which is 100 times the standard deviation divided by the mean (Goldstein, 1964). Use of this statistic permits one to consider the variation among the 3 counts independently of an effect of the magnitude of the counts, as shown by the regression of the C.V. on the total number of AFB:

$$
\text { C.V. } / 100=0.35-0.00012 \text { (no. AFB - 210). }
$$

The $95 \%$ confidence limits around the estimate of the slope of the regression line include 0 and the correlation coefficient, $r=0.12$, a value not different from 0 at 


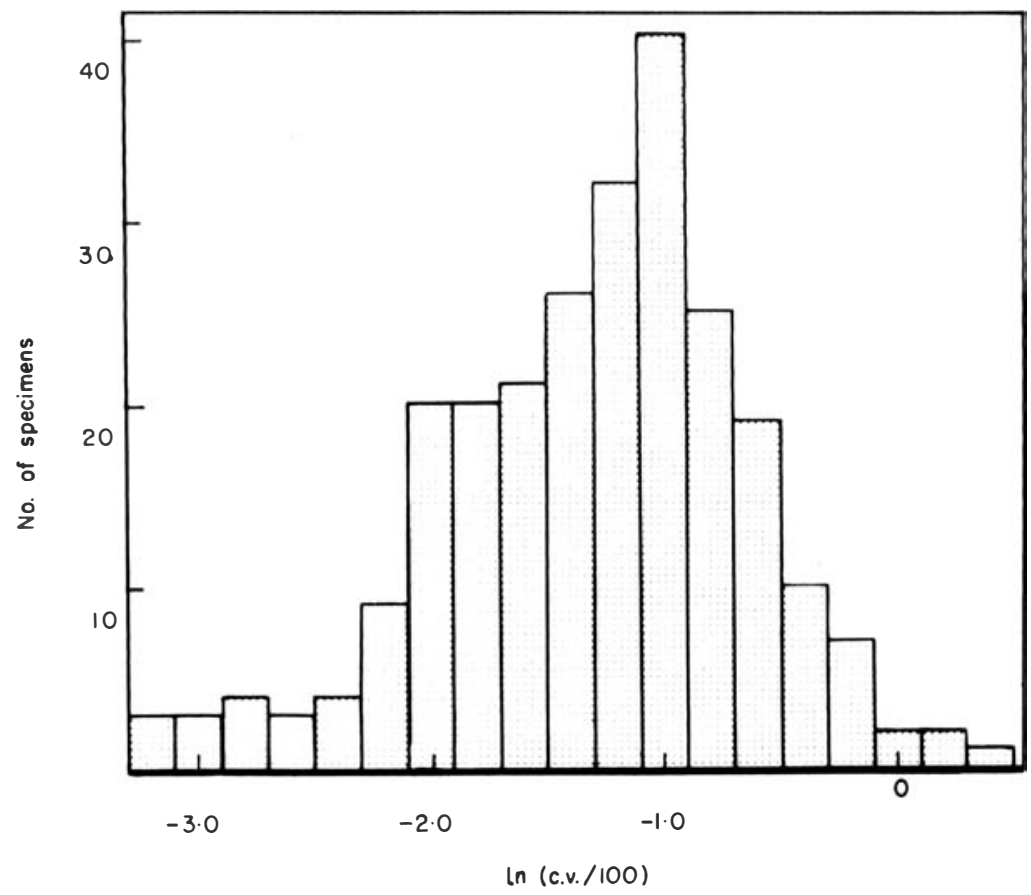

Fig. 3. Frequency distribution of $\ln (\mathrm{C} . \mathrm{V} . / 100)$ of the number of $\mathrm{AFB} /$ circle for 233 specimens.

$P=0.05$. Thus, the C.V. did not depend on the total number of AFB counted in the 3 circles of each slide.

The C.V. was not normally distributed, so that the dispersion of the individual values of the C.V. about the mean value could not easily be described. The natural logarithm $(\mathrm{ln})$ of the C.V. was distributed as shown in Fig. 3; the $\chi^{2}$ goodness-of-fit test showed that this distribution was consistent with a normal distribution $(P>0.1)$. The mean C.V. was $29 \%$, with $95 \%$ confidence limits of 26 and $31 \%$.

In 25 of the 233 slides ( $10.7 \%$ ), the number of AFB in 1 of of the 3 circles fell outside the range $-50 \%$ to $+100 \%$ of the mean for the 3 circles. These slicles yielded larger values of the C.V. Moreover, 14 of 77 slides yielding 50 to 99 $\mathrm{AFB} / 60$ fields were included in this group, whereas only 11 of the 156 slides with 100 or more AFB were included; these proportions are significantly different $(P<0.01)$. Thus, a disproportionately large fraction of these 25 slides was composed of slides with smaller numbers of AFB.

\section{VARIATION OF THE NUMBER OF AFB FROM FOOT TO FOOT}

On 13 occasions, harvests of $M$. leprae were performed from 4 individual foot-pads of mice of the same group. These results are summarized in Table 3. The ratio of the largest to the smallest number of AFB harvested from the 4 foot-pads of a group ranged from 1.67 to 20.0, with a mean of 6.66 . The mean C.V. for the 13 sets of 4 foot-by-foot harvests, calculated by averaging the values of 1 n C.V., was $60 \%$, with $95 \%$ confidence limits 45 and $79 \%$. 
TABLE: 3

Variation of the number of $A F B$ from foot to foot

\begin{tabular}{ccccccc}
\hline $\begin{array}{c}\text { Experiment } \\
\text { no. }\end{array}$ & \multicolumn{7}{c}{ Number of } & AFB/foot-pad $\left(\times 10^{5}\right)$ & Ratio & C.V.\% \\
\hline 1 & 1.38 & 3.60 & 8.89 & 13.1 & 8.49 & 78 \\
2 & 8.95 & 14.4 & 19.4 & 59.3 & 6.63 & 90 \\
3 & 1.90 & 7.27 & 11.5 & 12.4 & 6.53 & 58 \\
4 & 1.01 & 1.30 & 1.56 & 2.04 & 2.02 & 30 \\
5 & 3.77 & 4.63 & 5.69 & 10.6 & 2.81 & 49 \\
6 & 1.35 & 2.22 & 6.48 & 17.0 & 12.6 & 106 \\
7 & 0.85 & 1.02 & 3.92 & 6.00 & 7.06 & 84 \\
8 & 3.92 & 5.85 & 8.36 & 13.3 & 3.39 & 52 \\
9 & 0.70 & 0.84 & 0.84 & 1.55 & 2.21 & 39 \\
10 & 27.6 & 30.3 & 39.9 & 46.2 & 1.67 & 24 \\
11 & 2.21 & 2.88 & 5.11 & 13.3 & 6.02 & 87 \\
12 & 1.39 & 3.77 & 8.45 & 8.46 & 6.09 & 64 \\
13 & 0.28 & 1.70 & 2.79 & 5.61 & 20.0 & 87 \\
\hline
\end{tabular}

* Ratio of largest to smallest number in set of 4.

TABLE 4

Variation of the number of $A F B /$ foot-pad among replicate harvests from pools of 4 to 8 foot-pads

\begin{tabular}{|c|c|c|c|c|c|c|}
\hline \multirow{2}{*}{$\begin{array}{c}\begin{array}{c}\text { Experiment } \\
\text { no. }\end{array} \\
1\end{array}$} & \multicolumn{4}{|c|}{ Number of AFB/foot-pad ( $\left.\times 10^{5}\right)$} & \multirow{2}{*}{$\frac{\text { Ratio* }}{7.43}$} & \multirow{2}{*}{$\frac{\text { C.V. (\%) }}{57}$} \\
\hline & 0.85 & 4.22 & 4.70 & 6.34 & & \\
\hline 2 & 6.30 & 8.03 & 9.76 & 13.4 & 2.13 & 32 \\
\hline 3 & 2.04 & 2.07 & 2.24 & 4.80 & 2.35 & 48 \\
\hline 4 & 4.52 & 7.94 & 18.5 & 35.0 & 7.74 & 83 \\
\hline 5 & 2.38 & 3.32 & 5.07 & 6.94 & 2.92 & 45 \\
\hline 6 & 4.45 & 4.53 & 11.0 & 13.1 & 2.94 & 54 \\
\hline 7 & 0.17 & 0.50 & 0.50 & 0.68 & 4.00 & 46 \\
\hline 8 & 0.73 & 0.87 & 0.96 & 1.09 & 1.50 & 17 \\
\hline 9 & 4.47 & 5.17 & 6.26 & 7.31 & 1.64 & 21 \\
\hline 10 & 0.82 & 1.50 & 2.10 & 2.82 & 3.44 & 47 \\
\hline 11 & 13.00 & 15.0 & 19.0 & 32.0 & 2.46 & 43 \\
\hline 12 & 0.29 & 0.41 & 1.30 & 3.00 & 10.3 & 100 \\
\hline 13 & 0.88 & 0.91 & 2.10 & 2.39 & 2.72 & 50 \\
\hline 14 & 8.65 & 20.0 & 31.0 & 39.0 & 2.31 & 54 \\
\hline 15 & 0.12 & 0.19 & 0.60 & 0.88 & 7.33 & 80 \\
\hline 16 & 3.34 & 5.90 & 8.50 & 9.54 & 2.86 & 41 \\
\hline 17 & 1.05 & 1.35 & 1.56 & 1.87 & 1.78 & 24 \\
\hline 18 & 0.19 & 0.41 & 0.42 & 1.41 & 7.42 & 90 \\
\hline 19 & 3.20 & 5.92 & 11.2 & 11.3 & 3.53 & 51 \\
\hline 20 & 3.62 & 3.98 & 6.69 & 11.1 & 3.07 & 54 \\
\hline 21 & 5.62 & 15.6 & 21.7 & 22.5 & 4.00 & 48 \\
\hline 22 & 9.00 & 20.1 & 30.4 & 30.9 & 3.43 & 46 \\
\hline 23 & 0.85 & 1.50 & 2.53 & 3.74 & 4.38 & 59 \\
\hline
\end{tabular}

* Ratio of largest to smallest number in set of 4 . 


\section{VARIATION OF THE NUMBER OF AFB FROM HARVISST TO HARVI:ST}

On 23 occasions, 4 harvests of $M$. leprae were performed on the same day, each from the pooled tissues of 4 to 8 foot-pads of mice inoculated in the same experiment. The results of these harvests are presented in Table 4. The ratio of the largest to the smallest yield of the replicate harvests ranged from 1.50 to 10.3 , with a mean of 4.08 . The mean C.V. for the 23 sets of 4 replicate harvests, calculated by averaging the values of In C.V., was $48 \%$, with $95 \%$ confidence limits 40 and $57 \%$. Thus, the variation among replicate harvests of $M$. leprue from pools of 4 to 8 foot-pads is smaller than that among harvests from individual footpads, but greater than that found among circles prepared from the same bacterial suspension.

\section{DOUBLING TIME: OF: $M$. LEPRAE}

On many occasions during the past 8 years, 2 harvests of $M$. leprue were made during logarithmic multiplication of the same strain of organisms. On 129 occasions, the earlier of the 2 harvests yielded $>5 \times 10^{4}$ but $<5 \times 10^{5} \mathrm{AFB} /$ footpad. The slope of the line joining the 2 harvests was calculated for each of the 129 experiments, in order to derive an estimate of the doubling time of this strain of M. leprae during logarithmic multiplication. The mean slope was $0.094 \log ^{2}$ units (0.094 doublings)/day; the reciprocal of this slope represents a mean doubling time of 10.7 days. The $95 \%$ confidence limits around this estimate of the mean doubling time are 9.89 and 11.6 days per doubling.

\section{MULTIPLICATION IN BOTH HIND FEET COMPARED TO THAT IN ONE HIND FOOT}

In 8 experiments, mice of one group were inoculated RHF and mice of a second group were inoculated BHF with equal portions of the same bacterial suspension. Harvests of $M$. leprae from the pooled tissues of 4 foot-pads were performed at intervals, growth curves were constructed, and the number of days fron inoculation to multiplication to the level of $10^{6} \mathrm{AFB} /$ foot-pad was calculated from each growth curve. On the average, the time required for multiplication to $10^{6} \mathrm{AFB} /$ foot-pad of mice inoculated $\mathrm{BHF}$ was $6.5 \%$ longer than that of mice inoculated RHF; however, this value is not significantly different from 0 . The regression of the time to $10^{6} \mathrm{AFB} /$ foot-pad of mice inoculated BHF on that of mice inoculated RHF is shown in Fig. 4. The slope of the regression line, 1.41 , is significantly larger than 1.0 , indicating that multiplication of $M$. leprae is somewhat slower in mice inoculated BHF than in those inoculated RHF.

In order to interpret this finding, it is necessary to consider the possibility that fewer AFB were systematically inoculated LHF than RHF. In 16 experiments, mice that had been inoculated BHF were killed, and harvests of $M$. leprae were performed from separate pools of 4 RHF and 4 LHF. In 10 of these experiments, the numbers of AFB harvested from both pools were $>10^{5}$ and $<10^{6} /$ foot-pad. These data are presented in Table 5. The mean difference between the yields of $M$. leprae from pools of RHF and LHF was not significantly different from 0 . If the organisms may be assumed to have multiplied at the same rate in either hind foot-pad of mice inoculated in both, then there is no evidence that the number of $M$. leprae inoculated LHF was systematically different from that inoculated RHF. 


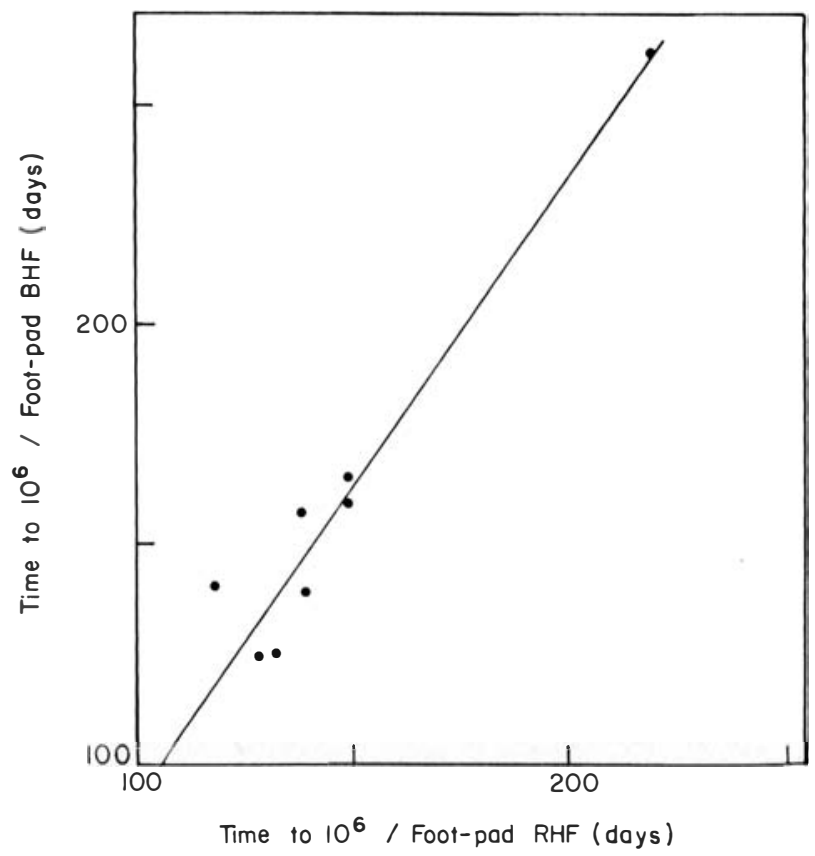

Fig. 4. Time to $10^{6} \mathrm{AFB} /$ foot-pad in mice inoculated $\mathrm{BHF}$ as a function of the time to $10^{6}$ $\mathrm{AFB} /$ foot-pad in mice inoculated RHF. The equation of the regression line is: No. days $\mathrm{BHF}=158+(1.41 \pm 0.29)($ No. days RHF -146$) ; r=0.98$.

TABLE 5

Variation of the number of $A F B /$ foot-pad from $L H F$ to $R H F$

\begin{tabular}{ccc}
\hline $\begin{array}{c}\text { Experiment } \\
\text { no. }\end{array}$ & $\begin{array}{c}\text { Number of AFB/foot-pad }\left(\times 10^{5}\right) \\
\text { RHF }\end{array}$ & $\begin{array}{c}\text { LHF } \\
\end{array}$ \\
\hline 1 & 5.79 & 4.12 \\
2 & 1.94 & 4.10 \\
3 & 3.93 & 2.27 \\
4 & 1.36 & 3.70 \\
5 & 9.16 & 6.84 \\
6 & 3.74 & 3.03 \\
7 & 9.70 & 5.27 \\
8 & 1.99 & 1.51 \\
9 & 4.62 & 2.86 \\
10 & 2.56 & 2.16 \\
\hline
\end{tabular}




\section{Discussion}

Application of Shepard's technique for cultivation of $M$. leprue in the mouse foot-pad (Shepard, 1960) have produced a considerable body of data that have subsequently been employed in making clinical decisions and in the selection of the drugs for trials and for use in therapy, to name but a few important uses. But because the technique is carried out in only a few laboratories, many workers who use its results do not understand clearly its strengths and limitations. The performance of the technique during the 10 years since this laboratory was established has generated much data, an analysis of which may be informative to some of the "consumers" of these and similar data. The purpose of this report is to present such an analysis.

The presentation is divided into 6 sections. The first 4 sections represent a progressive increase of scale, beginning with the distribution of AFB among the microscopic fields examined in a circle of a counting slide, and ending with the reproducibility of the harvest procedure performed on the pooled tissues of a number of mouse foot-pads. The last 2 sections represent "by-products" of this analysis - a second look at the doubling time of $M$. leprae, and a comparison of the rate of multiplication in mice inoculated in both hind foot-pads to that in animals inoculated in one.

In the performance of Shepard's technique, AFB are enumerated in only a minute fraction of the bacterial suspension that results from a harvest of $M$. leprae. A $10-\mu \mathrm{l}$ aliquot of a suspension $2 \mathrm{ml}$ or greater in volume is pipetted onto each of the surfaces bounded by the 3 circles of a counting slide, and AFB are enumerated in $201250 \times$ oil-immersion fields along the equator of each circle. The diameter of a $1250 x$ field of one of our microscopes is $0.18 \mathrm{~mm}$, and that of a circle of a slide from the batch currently in use is $1.13 \mathrm{~cm}$. Therefore, one field represents 0.00025 of the area of a circle, or $0.0025 \mu \mathrm{l}$, and the 60 fields examined represent $0.15 \mu \mathrm{l}$, or 0.00075 of a $2-\mathrm{ml}$ suspension. Had we established that the AFB were independent and randomly distributed, in the sense that their distribution fit a Poisson distribution, then accurate estimates of the number of AFB in the mouse foot-pad could be readily obtained, the only source of variation being sampling (experimental) error. However, the results indicate that one cannot assume the AFB to be randomly distributed on the circles of a counting slide, especially when the numbers of AFB are large.

Such a situation could have resulted from "clumping" of the $M$. leprae, both as closely-grouped organisms and as groups of well-separated organisms contained within tissue cells that had escaped disruption. These phenomena, encountered not infrequently in work with mouse foot-pad technique as described by Shepard (1960), would produce more fields containing both larger and smaller numbers of AFB than would be predicted from the mean number of AFB per field. The demonstration that a non-random distribution was associated with larger numbers of organisms is difficult to interpret. Even a small value of $\lambda$, the mean number of organisms/oil-immersion field, is consistent with multiplication of $M$. leprae. Specimen no. $8(\lambda=2.0)$, for example, represented a yield of $5.62 \times 10^{5} \mathrm{AFB} /$ foot-pad, more than 100 times the number of $M$. leprae inoculated. Thus, the association of non-randomness with larger numbers is not simply a result of multiplication.

Because it is not possible to quantitate the increased variation that results from the non-random distribution of the AFB, other methods of assessing the precision 
of the mouse foot-pad technique must be employed. The C.V. offers another means of assessing variation in a population. Although it may lack a degree of precision, it possesses the advantages of independence of the size of the population-in this case, the number of AFB counted. And we have shown that the $\ln$ C.V. is approximately normally distributed, permitting the application of normal statistics for testing the hypotheses and determination of confidence intervals. The usefulness of the C.V. resides in its relationship to the standard deviation. If the mean number of AFB counted in the 3 circles of a counting slide is 100, then the "true mean" lies in the range $100 \pm 29$ (mean $\pm(C . V . / 100) \times$ mean $)$, and the $95 \%$ confidence limits around the number of $\mathrm{AFB} /$ foot-pad calculated from the mean or total number of AFB counted in 3 circles would be $29 \%$ larger and $29 \%$ smaller than the number calculated.

One might expect that the C.V. for replicate slides would be smaller than that for replicate circles-i.e. that the variation of the number of AFB between duplicate slides would be smaller than that among triplicate circles, and that the C.V. for 2 repeated counts on the same slide would be smaller yet. In the course of another study (Levy et al., 1976), 2 observers performed duplicate counts on 20 slides. The mean values of the C.V. calculated for each observer were 9 and $14 \%$; the mean ratios of the larger to the smaller number of $M$. leprue per slide were 1.33 and 1.31 , respectively. These values are significantly smaller than those for replicate circles.

Although $M$. leprae are most often harvested from pools of mouse foot-pad tissues rather than from the tissues of individual foot-pads, the variation of the number of $\mathrm{AFB}$ from foot to foot was of some interest. Harvests from sets of 4 foot-pads yielded a C.V. of $60 \%$ and a mean ratio of the largest to the smallest

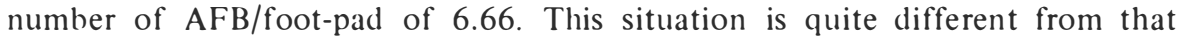
representing more common application of foot-by-foot harvesting, in which one seeks evidence of variation among animals or evidence that a marginally adequate inoculum has infected some animals but not others. In these latter applications, one would expect a much larger value of the C.V., with a much broader range between the smallest and largest number of $\mathrm{AFB} /$ foot-pad.

One would also expect that the variation among harvests of $M$. leprae from replicate pools of mouse foot-pad tissues would be smaller than that among individual foot-pads. This was indeed the case; the C.V. is smaller for harvests from replicate pools of from 4 to 8 foot-pads than for harvests from individual foot-pads. A most important observation is that pooling the tissues does not, under ordinary circumstances, obscure really unacceptable variation from foot to foot.

One by-product of this study was the measurement of the doubling time of a single strain of $M$. leprae during logarithmic multiplication in mice. In another study (Levy, 1976), we found the doubling time, measured by a different technique, to be $11.1 \pm 1.9$ days. The measurement made in this study $-10.7 \pm 0.8$ days-is virtually identical.

A second by-product was the comparison of rates of multiplication of $M$. leprae in mice inoculated BHF to that in mice inoculated only RHF. Multiplication in animals inoculated BHF was significantly slower, but the difference of rates was so small that it was not meaningful biologically. The difference did not reflect a systematically smaller inoculum LHF. This observation is of some practical importance. In many experiments we inoculate mice BHF rather than RHF in order to economize on mice, cages and the demands on the staff of our animal 
house. We had been reassured that this was an acceptable procedure by the results of an earlier study (Levy, 1975), in which we showed that mice infected with $M$. leprae in one hind foot-pad 15 to 60 days earlier were not protected against $M$. leprae challenge in the second hind foot-pad. At every challenge interval, multiplication of $M$. leprae in the challenge inoculum was compared to that in control mice not previously infected. The control mice were inoculated BHF. Perhaps had the control mice been inoculated RHF, we might have observed a small effect of the primary infection at the earlier intervals similar to the differences observed in the current study between mice inoculated BHF and those inoculated RHF.

The dat a presented in this analysis suggest that the mouse foot-pad technicue as described by Shepard exhibits a considerable degree of precision. Except in the case of foot-by-foot harvests, differences among measurements equivalent in time or in numbers of $M$. leprae to at least one doubling (or halving) may be accepted as real; differences equivalent to two doublings appear certainly to be meaningful.

\section{Acknowledgements}

This research was supported in part by the U.S. Leprosy Panel of the U.S.-Japan Cooperative Medical Science Program administered by the Geographic Medicine Branch, National Institute of Allergy and Infectious Diseases, National Institutes of Health, Bethesda, Maryland 20014, U.S.A. (Grant R22 AI 07801).

\section{References}

Goldstein, A. (1964). Biostatistics. New York: Macmillan.

Levy, L. (1975). Superinfection in mice previously infected with Mycobacterium leprae. Infect. Immun. 11, 1094.

Levy, L. (1976). Studies of the mouse foot-pad technique for cultivation of Mycobacterium leprae. 3. Doubling time during logarithmic multiplication. Lepr. Rev. 47, 103-106.

Levy, L., Hom, C. and Murray, L. P. (1976). Enumeration of Mycobacterium leprae stained with and without prior periodate oxidation. Lepr. Rev. 47, 185-191.

Levy, L., Moon, N., Murray, L. P., O’Neill, S. M., Gustafson, L. E. and Evans, M. J. (1974). Studies of the mouse foot-pad technic for cultivation of Mycobacterium leprae. 1. Fate of inoculated organisms. Int. J. Lepr. 42165.

Levy, L. and Murray, L. P. (1976). Studies of the mouse foot-pad technique for cultivation of Mycobacterium leprae. 2. The relationship between incubation period and generation time. Lepr. Rev. 47, 13.

Shepard, C. C. (1960). The experimental disease that follows the injection of human leprosy bacilli into foot-pads of mice. J.exp. Med.112, 445 .

Shepard, C. C. and McRae, D. H. (1968). A method for counting acid-fast bacteria. Int. J. Lepr. 36,78 . 